\title{
Solar Meridional Flows: Recent Findings
}

\author{
Deborah A. Haber \& Bradley W. Hindman \\ JILA and Dept. of Astrophysical and Planetary Sciences, University of \\ Colorado, Boulder, CO 80309-0440, USA
}

\begin{abstract}
We report new measurements of the sun's global meridional circulation within the near-surface shear layer for the years 1996 to 2003 . The flows are obtained using the local helioseismic technique of ring analysis applied to MDI Dynamics Program data. The most recent estimates of the solar p-angle correction to this data have been incorporated. Previously published accounts that the solar meridional circulation possesses multiple cells within the northern hemisphere (Haber et al. 2002) are not contradicted by the new findings. We do find, however, that with the inclusion of the p-angle correction, cross-equator flows have been eliminated.
\end{abstract}

\section{Introduction}

The meridional circulation deduced using local helioseismology techniques such as ring analysis and time-distance tomography reveals asymmetries between the northern and southern hemispheres (Giles 1999; Haber et al. 2000, 2002; Zhao $\&$ Kosovichev 2003). The asymmetries manifest themselves as a southward flow across the equator on the order of $6 \mathrm{~m} \mathrm{~s}^{-1}$, as multiple meridional cells in the northern hemisphere from 1998 to the present, or as faster flows in the southern hemisphere.

There has been general agreement that a southward flow across the equator is seen in the MDI data (e.g., Schou \& Bogart 1998; Basu \& Antia 2000; Haber et al. 2000, 2002). However, some researchers have suggested that this crossequator flow arises from a misidentification of the Sun's rotational axis. There are not any fundamental theoretical reasons why the meridional flow should vanish at the equator. But the orientation of the MDI CCD chip with respect to the solar north pole is only known to a few tenths of a degree. A small error in the measurement of the p-angle can lead to mixing of the large differential rotation signal into what is identified as meridional flow.

Haber et al. (2002) and Giles (1999) both saw reversal of the meridional flow in a submerged zone in the northern hemisphere starting in 1998, while in the southern hemisphere the meridional flow remained polewards at all depths. This finding has not been universally reported (Chou \& Dai 2001). These discrepant findings may result from the use of instruments with different (or minimal) pangle errors, or they may arise from differences in the depth dependence of the averaging kernel sensitivities.

\section{Meridional Flow Observations With and Without Corrections}

There now exist measurements of the MDI p-angle offset acquired by crosscorrelating MDI data with coeval GONG data whose p-angle was calibrated using Mercury transits of the Sun (Toner 1999). The results of these comparisons 
have shown that the north pole of the MDI images diverges from the nominal solar rotation axis by $0.2^{\circ}$, yielding an error of $\sim 7 \mathrm{~ms}^{-1}$ in the meridional flow. An additional effect that should be considered is an error in the determination of the Carrington elements. Giles (1999) performed a fit to time-distance measurements of the meridional flow for two years of MDI data and discovered that $\Delta i=-(0.091 \pm 0.012)^{\circ}$ and $\Delta \Omega=-(0.18 \pm 0.10)^{\circ}$ where $i$ is the inclination of the solar equator to the ecliptic and $\Omega$ is the longitude of the ascending node of the solar equator on the ecliptic plane.

The observations reported here were generated using the ring analysis technique described briefly in Hill's review of local helioseismology appearing in this volume (2003) and in Haber et al. (2002). Figure 1 shows the longitudinally and temporally averaged meridional flows at a depth of $11.6 \mathrm{Mm}$ for the years 1996 to 2003. Both the original processing of the full MDI Dynamics Programs

\section{Meridional Velocity $\left(\mathrm{ms}^{-1}\right)$}
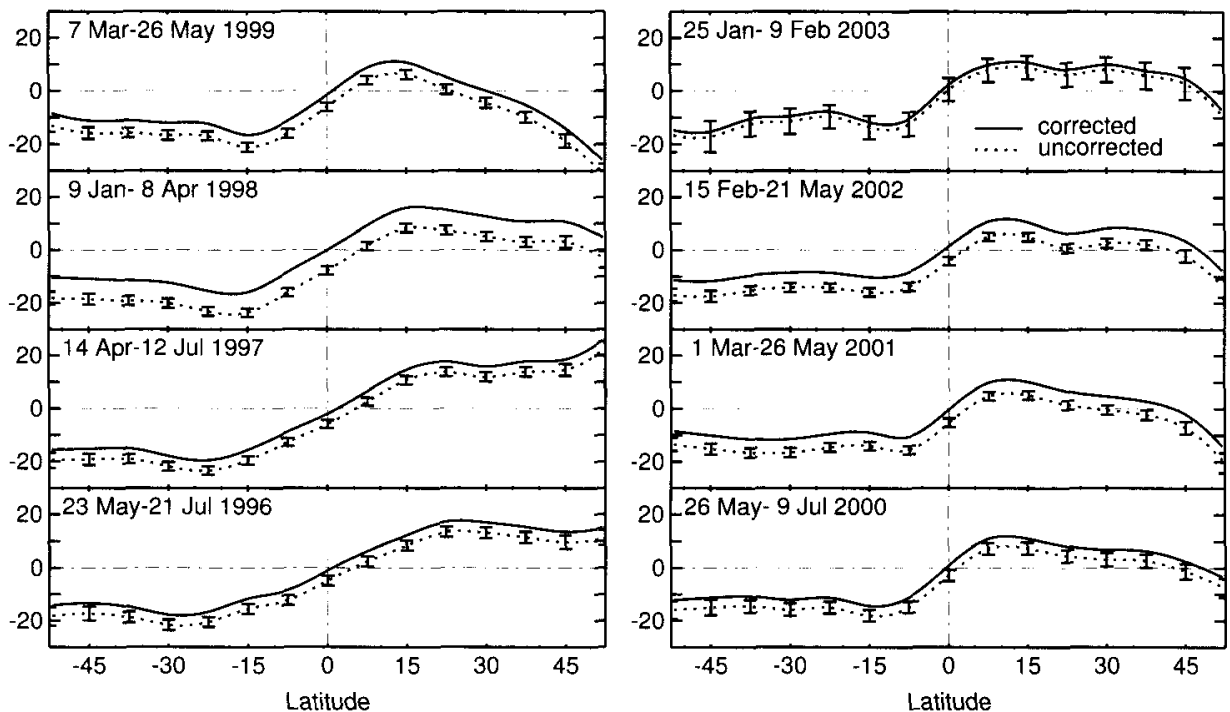

Figure 1. Mean meridional flows determined from longitudinal and temporal averages over the dense-pack mosaic for the full MDI Dynamics period from each of the past 8 years for the uncorrected data (dashed line) and the corrected data (solid line). The results of the inversions at a depth of 11.6 $\mathrm{Mm}$ are shown with $10 \sigma$ error bars, where $\sigma$ is the formal error. Notice that the velocity at the equator tends to be smaller for the corrected data and that the latitude at which the meridional flow becomes negative in the northern hemisphere is higher than in the uncorrected case.

and the same data corrected for the p-angle and possible misidentification of the Carrington elements are shown. Figure 2 presents $(a)$ the uncorrected and $(b)$ the corrected meridional circulation over the years 1998 to 2003 as a function of latitude and depth. The extent of the additional meridional cell is more clearly portrayed in this representation. We see that the submerged meridional cell with reversed flow in the northern hemisphere still exists after the data has been 

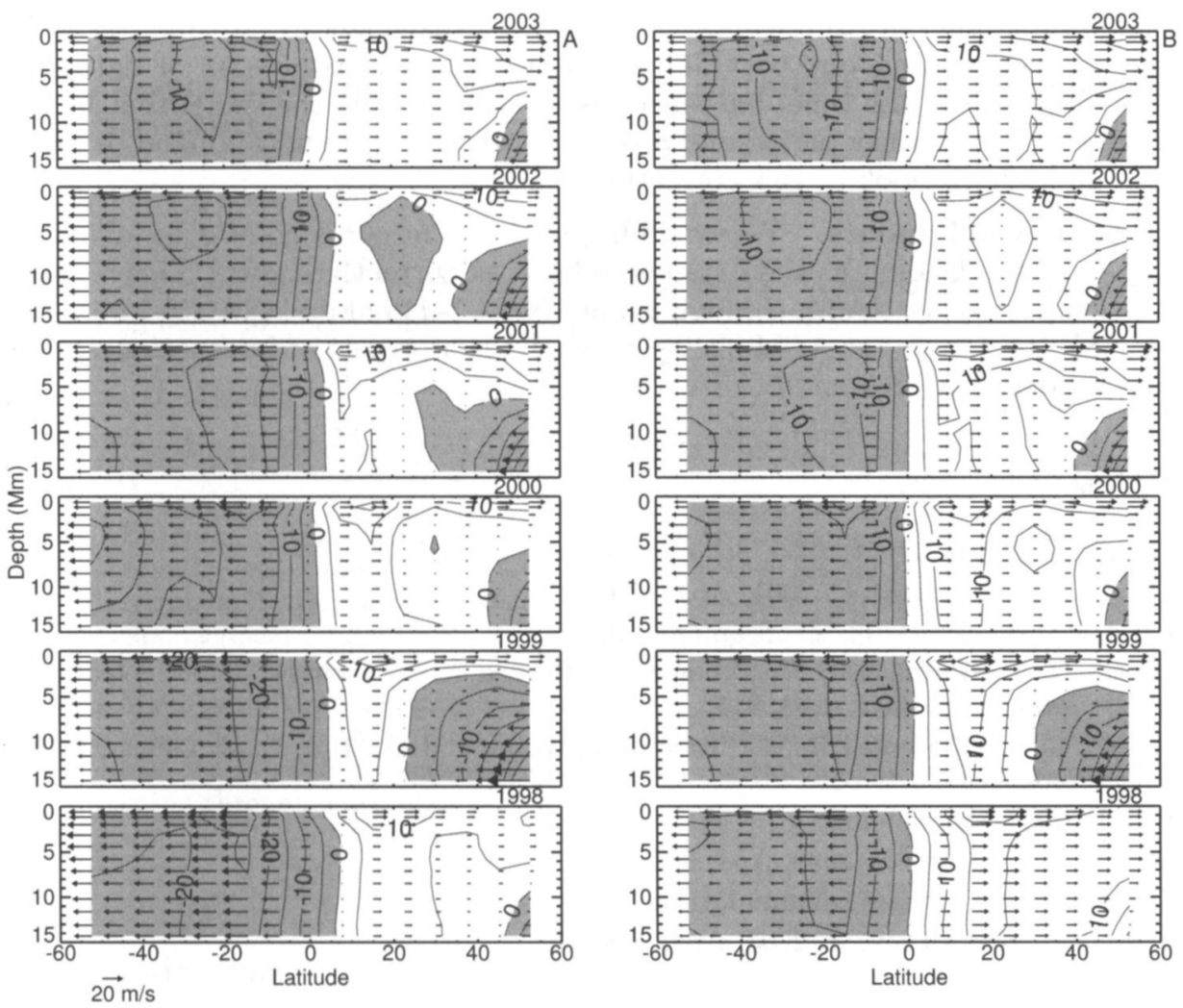

Figure 2. The longitudinally and temporally averaged meridional flow as a function of latitude and depth: $(a)$ uncorrected and $(b)$ corrected for the misidentified p-angle and Carrington elements. The meridional flows are indicated with both contours and arrows, with the negative contours corresponding to southerly flow (shaded in grey). The submerged cell of reversed circulation in the northern hemisphere appears in both the corrected and uncorrected data.

corrected. Albeit this cell appears at greater depths and higher latitudes than previously reported. In any case, the meridional flow in the northern hemisphere became very different from that in the southern hemisphere as magnetic activity rose with the advancing solar cycle.

\section{Discussion}

It is clear that with the corrections as they are now known, the submerged cell in the northern hemisphere is still present and the meridional flow at depth in this hemisphere is still much less uniform than it is in the southern hemisphere. The southward flow across the equator, however, is almost completely eliminated.

These corrections, however, are still preliminary. The correction of the MDI p-angle has so far been analyzed for only two days of data spaced 6 months apart in 1999. Both measurements gave a value of $0.2^{\circ}$ but it would be useful to 
analyze the other measurements that have been taken since that time. Likewise, the analysis carried out by Giles (1999) was for the two years of data that were available at the time, but since (as he pointed out) the signal that he was analyzing has a yearly period and 8 years of MDI data are now in hand, it would also be useful to look at this correction again.

Acknowledgments. We would like to thank Jesper Schou for useful discussions and for sending us the data corrections as currently known. This research was supported by NASA through grants NAG 5-11920 and NAG 5-12491 and by NSF through grant ATM-0219581. SOHO is a project of international cooperation between ESA and NASA.

\section{References}

Basu, S. \& Antia, H. M. 2000, Solar Phys., 192, 469

Chou, D.-Y. \& Dai, D.-C. 2001, ApJ, 559, L175

Giles, P. 1999, Ph.D. Thesis, Stanford University

Haber, D. A., Hindman, B. W., Toomre, J., Bogart, R. S., Thompson, M. J., \& Hill, F. 2000, Solar Phys., 192, 335

Haber, D. A., Hindman, B. W., Toomre, J., Bogart, R. S., Hill, F., \& Larsen, R. M., 2002, ApJ, 570, 855

Hill, F. 2003, this volume

Schou, J. \& Bogart, R. 1998, ApJ, 504, L131

Toner, C. 1999, private communication

Zhao, J. \& Kosovichev, A. 2003, ApJ, 591, 446 